\title{
Keloids: current prophylactic and therapeutic methods
}

\author{
Wojciech Bienias, Andrzej Kaszuba
}

Department of Dermatology, Pediatric Dermatology and Dermatological Oncology, Medical University of Lodz, Poland Head: Prof. Andrzej Kaszuba MD, PhD

Postep Derm Alergol 2012; XXIX, 6: 446-450

DOI: 10.5114/pdia.2012.32392

\begin{abstract}
Prophylaxis and therapy of keloids present a big challenge for medicine, despite the increasing knowledge about their pathogenesis. There are a lot of therapeutic methods but their effectiveness is still limited and unsatisfactory. These methods are divided into surgical, pharmacological and physical ones as well as the most frequently applied combined therapy, which is the most effective one. A surgical way of removing keloids is becoming less common and the method of intralesional application of steroids still remains the treatment of choice. In addition to the above there are cryotherapy, laser therapy, pressure therapy, X-ray irradiation and silicone products in a form of gels and dressings. Relatively new methods are intralesional injections of verapamil and 5-fluorouracil. Enalapril and troglitazone, which are currently used in the treatment of hypertension and diabetes, are expected to be of use as well. Biological drugs like monoclonal antibodies against tumor growth factor (TGF)- $\beta 1$, TGF- $\beta 2$ and vascular endothelial growth factor and analogues of TGF- $\beta 3$ are also potential solutions in the treatment of keloids. Further studies on the treatment of keloids should result in more efficient therapies.
\end{abstract}

Key words: keloids, surgery, glucocorticosteroids, cryotherapy, laser treatment, pressure therapy, X-ray irradiation.

\section{Introduction}

Keloids present a great challenge in prophylaxis and therapy. Despite the fact that many drugs and therapies are available nowadays, there is no fully effective or satisfying method to deal with that disorder [1-6].

A considerably growing interest in the pathogenesis of keloids as well as increasing knowledge on the problem resulted in the development of more efficient and promising ways of eliminating this frustrating disease, which is partly dealt with by dermatology, plastic surgery or aesthetic medicine. Bearing in mind the fact that many previously applied methods turned out to be failures, we should remember the famous medical slogan, which says that "it is easier to prevent diseases from developing rather than treat them after they have progressed". With regard to keloids, this saying becomes really meaningful [1-7].

\section{Prophylactic methods}

The most important factor in the prophylaxis of keloids is a detailed anamnesis which focuses on genetic condi- tions for developing keloids, including a detailed analysis of potential genetic predispositions as well as anamnesis concerning some concomitant diseases (scleroderma, Rubinstein-Taybi syndrome, progeria) and even a blood group. They are all aimed at identifying a patient who demonstrates a higher risk of developing keloids. The patient should avoid any injuries or surgeries which break skin integrity, especially, incisions, piercing, tattoos and even unnecessary injections or vaccination. Such diseases like furuncle, acne or herpes, disorders which are commonly observed and usually easy to treat, in patients who are predisposed to keloids should be treated immediately, as they might transform just into keloid forms. If it happens, the patient should be treated with care; topical or even systemic antibiotic therapy is advisable. If the patient has to undergo an operation, surgical incisions should be minimal in size and number. They should follow skin tension lines and be made in places which are most affected by keloids. Doctors also perform plastic surgeries which reduce skin tension and use self-absorbing stitches. After the surgery the incision area should be properly taken care of [1-10].

Address for correspondence: Wojciech Bienias, Department of Dermatology, Pediatric Dermatology and Dermatological Oncology, Medical University of Lodz: 1/5 Kniaziewicza St, 91-347 Lodz, Poland, phone: +48 42651 97 54, e-mail: wojtekb82@buziaczek.pl 


\section{General principles of the therapy of keloids}

If the patient has developed a keloid, despite having made efforts to prevent it, doctors should immediately initiate a treatment session. The first principle says that the older the keloid is, the more resistant to treatment it becomes. Thus, a therapy should start soon after the diagnosis has been set. According to another principle, doctors should apply a few therapeutic methods simultaneously, i.e. use a combined therapy as monotherapy is usually not highly effective [1-11]. A keloid therapy must be regular and systematic. One surgery is never enough. Sometimes a patient must undergo a few or a dozen therapeutic sessions and wait for effects for a long time. Therefore, he should be made aware of all this and be properly motivated to be willing to undergo the therapy. Otherwise, he will give it up soon without showing any considerable improvement [1-12].

The patient should also be informed that the keloid will not disappear without leaving any traces and the therapy is aimed (currently) at making it look as good as possible, reducing the volume of the keloid and its consistency. Thanks to such improvements, the patient is more prone to accept the disease. In the case of keloids which have spread extensively and cover a large area of the patient's body, in visible and open places, the therapeutic process should be dealt with a psychologist, psychotherapist and sometimes, a psychiatrist [1-12].

Another principle involves a detailed observation of therapeutic effects and also adverse effects and complications. They will be described further in the paper.

\section{Common therapeutic methods}

The methods are divided into surgical, pharmacological and physical ones. The surgical method has been used for a long time and it includes removal of the keloid with some skin margin. Keloids are defined as disorders which recur after they have been removed and new keloids are even bigger than the former ones. The recurrence percentage ranges from $40 \%$ to $100 \%$. Thus, this method has been considered ineffective and even contraindicated in the therapy of keloids. The only exception is combining this method with $\mathrm{X}$-ray irradiation and pharmacological methods with the use of appropriate surgeries and atraumatic stitches. The risk of complications is however high. Thus, doctors should carefully consider indications for performing that kind of treatment [1-14].

Another method - intralesional steroid therapy - has also been used for some time and still remains a selected therapy. Triamcinolone acetate was the most commonly applied preparation. It was administered at a dose of $40 \mathrm{mg} / \mathrm{ml}$. Currently, it has been banned on the pharmaceutical market. Slow-release steroids are now more preferred. The efficacy of the intralesional steroid therapy is up to $72 \%$ (the look of the keloid is much improved) and around $64 \%$ (the keloid area is completely smooth). The injection should be carried out with the smallest possible needles every 4-6 weeks. At the same time the doctor should observe the patient carefully and trace any possible complications, which include skin atrophy, telangiectasia, hyperpigmentation or hypopigmentation. After rejecting the injection sessions, the possibility that the keloid will recur within a period of 5 years is $50 \%[1-10,15,16]$. Another method is an intralesional injection with 5-fluoroacil. It is an antimetabolite used in neoplastic therapy and precancerous skin conditions. Its antimitotic activity is used in a keloid therapy. A dose of 50-150 mg in one injection is administered and topical irritation is the major adverse effect $[1-11,17,19]$. The keloid can be also treated with injections consisting of steroid and 5-fluoroacil. These two combined drugs appear to bring better results rather than when they are administered separately [16].

Laser therapy is another option in the treatment of keloids. In the past doctors used mainly $\mathrm{CO}_{2}$ laser but it did not turn out to be fully effective [1-11, 20, 21].

Currently, a pulse dye laser (PDL laser), which emits a beam of $585 \mathrm{~nm}$, is considered the most effective one in the keloid therapy. Its efficacy is $70 \%$. The target of the laser beam is hemoglobin present in blood cells which fill the lumen of the new vessels of the keloid. The energy which is released, destroys the vessels but does not destroy any surrounding structures. There is, however, a disadvantage. Melanin present in the epidermis has a similar absorption spectrum. It thus weakens the laser beam. Before it gets to the target, i.e. the vessels, it is partly absorbed by the epidermis. Here, it should be emphasized that people with darker skin, that is those who have more melanin, more often develop keloids. Laser procedures should be applied every 3-6 weeks. Hyperpigmentation is a typical adverse effect, which however, appears rarely. The procedures are painless and do not take much time. They are also quite safe [20, 21].

Application of dressings and silicone gels are other ways of prevention of keloids. Occlusion appears and the skin becomes more hydrated. Keratinocytes are stimulated to create and secrete necessary cytokines and growth factors [1-11, 22-26].

The dressings and silicone gels should be applied topically, which means that they are put on the keloid and left for at least $12 \mathrm{~h}$ every 4-6 weeks. Side effects include mainly skin maceration and slight skin irritations. The efficacy of the method is high - up to $80 \%$. It is inconvenient and causes discomfort for the patient. In consequence, he is not really willing to comply with recommendations or is not consistently following the therapy [1-11, 22-26].

Pressure therapy, i.e. therapy which uses pressure, is another therapeutic option. A simple mechanism is used here. The pressure inhibits perfusion, which leads to hypoxia, cell metabolism slowing down, fibroblast proliferation and collagen production. The pressure must be however controlled so as not to cause necrosis of healthy tissues. In the keloid therapy, doctors use pressure of 20-40 mm Hg, depending on the location of the keloid and its size. It is 
advisable to use the therapy throughout day and night. This method is frequently used to treat keloids which have appeared on earlobes. Some pressure clips are put on the patient's ears. The clips resemble clips which are commonly worn as jewellery. In the case of keloids localized on the trunk or arms, doctors can put special pressure garments. They are mainly useful in the therapy of post-burn keloids. Scientists still do not know much about the effectiveness of pressure therapy in the treatment of keloids. This kind of therapy is inconvenient and causes a lot of discomfort, which is a great disadvantage. Patients hardly ever consistently comply with its requirements [1-11, 27].

Radiotherapy, or topical application of X-radiation is another therapeutic option, however less and less frequently used. It is mainly used in prophylaxis and is associated with surgical methods. Already on the day following the surgery, the area which was incised, is irradiated with doses of 10-25 Gy, depending on the localization of the lesion. The healthy skin is protected against radiation with special filters. Radiotherapy is connected with many adverse effects, starting from erythema and desquamation to hyperpigmentation, telangiectasia and even skin atrophy. With regard to irradiation of existing keloids, scientists are still unsure whether or not this X-radiation therapy transforms lesions into their malignant forms.

In prophylaxis doctors apply a few sessions, gradually increasing the dose [1-12, 14, 28].

Another therapy, a physical one, is cryotherapy. This method is commonly used in various skin disorders, such as skin cancers, premalignant conditions or virus warts. Here special caps are used. First the temperature of those caps is lowered in liquid nitrogen to $-180^{\circ} \mathrm{C}$. Then the caps are placed on the keloid area for 10-30 s. Then the tissue gets defrosted within a period of time 2-3 times longer than the freezing time. The cycle of freezing and defrosting can be applied not more than twice during one procedure. They are administered at 1-month intervals. The effectiveness of this method is $50 \%$ with regard to the size of the keloid. The effect is even more visible when the initial size of the keloid is not so big.

Side effects of this method include hypopigmentation in the area of the surgery and post-surgical pain [1-12, 29-31].

The methods described above are standard ones. Some of them are becoming less and less important. In contrast, some others become more popular than before.

\section{Therapeutic novelties}

$5 \%$ Imiquimod in the form of cream has been recently used in the keloid therapy. It is used topically. Before, it was applied to treat pre-cancerous conditions or superficial basal cell carcinoma. The mechanism of activity includes stimulation of the production of mediators such as interferon (IFN) or tumor necrosis factor (TNF) as well as some other cytokines which inhibit fibrosis and prolif- eration of fibroblasts. Side effects occur infrequently and they include erythema and irritation. The effect of the therapy appears to be short-term if the drug is stopped being administered, which makes the therapy disadvantageous [1-9, 32].

A relatively recent pharmacological method is intralesional injections with verapamil administered at a dose of $2.5-5 \mathrm{mg} / \mathrm{ml}$. Verapamil is a drug from a group of calcium channel blockers, so far used in the therapy of common diseases such as arterial hypertension or supraventricular arrhythmia. The mechanism of activity of verapamil includes a decrease in the amount of ionized calcium in fibroblast cytoplasm. As a result, fibroblast proliferation is reduced and so is the production of collagen [7-11, 33, 34].

Since it is a relatively recent therapeutic method, the evaluation of its effectiveness cannot be fully reliable. However, it is estimated that the percentage is $50 \%$. At the same time hardly any side effects were observed [33, 34].

Many professional studies on keloid therapies contain information on the application of bleomycin, penicillamine, retinoids, colchicine and even botulinum toxin type $A$. The effectiveness of many of these preparations is minimal and the mechanism of activity - vague. Thus, the authors of this study think it is difficult to evaluate the real role of the drugs in the keloid therapy $[1-12,35,36]$.

A constant development of the knowledge of keloid pathogenesis contributes to adding some new drugs to thelist, which is constantly growing [36].

Enalapril is a drug which will probably be soon entered into the list. It is a hypotensive drug and an inhibitor of angiotensin converting enzyme. Enalapril has been applied in the therapy of arterial hypertension for a long time and appears to be highly effective. Drugs from this group, however, not only reduce arterial hypertension. It was confirmed that inhibitors of angiotensin converting enzyme play a positive role in post-infarction remodeling of the heart wall, in the mechanism of fibrosis inhibition and scarring of the cardiac muscle. It was also proved that they inhibit lung fibrosis, which depends on tumor growth factor (TGF)- $\beta$ signaling path [37].

So far, researchers have given an account of 2 patients with keloids who, for cardiologic indications, were administered enalapril at a dose of $10 \mathrm{mg}$ for a period of 4 months. After that time they noted a beneficial influence of the drug and a substantial improvement of the keloid; it got reduced in size and its consistency also changed. Detailed studies on the application of the drug (or a few drugs) in the therapy and prophylaxis of keloids seem to be fully justifiable and the drug is expected to bring beneficial effects [37].

Troglitazone is another drug, which might be potentially effective in prevention and the therapy of keloids. It is an anti-diabetic drug, PPAR receptor agonist. Molecular studies on diabetes with the use of rats' pancreases confirmed that troglitazone inhibits TGF- $\beta 1$ - SMAD 2 and 3 signaling path. Since it is also the main path promoting fibrosis in keloidogenesis, the results of the studies made the 
researchers analyze the effect of troglitazone on keloid fibroblasts in vitro. As it had been expected, troglitazone added to the fibroblast cultured from keloids considerably contributed to a decrease in the concentration of all transmitters of the path as well as the final product - procollagen 1. Until now the efficacy of troglitazone in patients with keloids has not been studied. Nevertheless, the results of some molecular studies clearly show that such studies should be conducted. Troglitazone might contribute to lengthening the list of anti-keloid drugs [38].

\section{Therapies of the future}

In the time of biological therapy, which is becoming more and more common, monoclonal antibodies against TGF- $\beta 1$ and TGF- $\beta 2$, TGF- $\beta 3$ analogue and monoclonal antibody against vascular endothelial growth factor (used in the therapy of some cancers of large intestine and in the exudative form of AMD) are used [1-12, 39].

Thanks to rapid advancement of genetic engineering and further development of the knowledge on particular genes and their mutations, which contributes to keloidogenesis, scientists should be able to find some gene repairing properties [40].

Researchers hope that the knowledge on the pathogenesis of keloids and further studies on drugs which might be introduced in the therapy of keloids, will make this therapy fully effective and satisfying for patients.

\section{References}

1. Jabłońska S, Majewski S. Skin diseases and sexually transmitted diseases [Polish]. PZWL, Warsaw 2008.

2. Sterry W, Paus R, Burgdorf W. Dermatology [Polish]. Czelej, Lublin 2009

3. Braun-Falco, Burgdorf WHC, Plewig G, et al. Dermatology. Part I [Polish]. Czelej, Lublin 2010.

4. Kaszuba A, Adamski Z. Dermatology lexicon. Part I [Polish]. Czelej, Lublin 2011.

5. Al-Attar A, Mess S, Thomassen JM, et al. Keloid pathogenesis and treatment. Plast Reconstr Surg J 2006; 117: 286-94.

6. O'Sullivan ST, O'Shaughnessy M, O'Connor TPF. Aetiology and management of hypertrophic scars and keloids. Ann R Coll Surg Engl 1996; 78: 168-75.

7. Slemp EA, Kirschner E. Keloids and scars: a review of keloids and scars, their pathogenesis, risk factors and management. Curr Opin Pediatr 2006; 18: 396-402.

8. Chike-Obi CJ, Cole PD, Brissett AE. Keloids: pathogenesis, clinical features and management. Semin Plast Surg 2009; 23: 178-84.

9. Bienias W, Miękoś-Zydek B, Kaszuba A. Current views on the etiopathogenesis of keloids. Postep Derm Alergol 2011; 28: 467-75.

10. Zieliński T, Witmanowski H, Lewandowicz E, et al. Hypertrophic scars and keloids. Part I - Prevention and treatment [Polish]. Postep Derm Alergol 2008; 25: 116-24.

11. Davidson S, Aziz N, Rashid RM, Khachemoune A. A primary care perspective on keloids. Medscape J Med 2009; 11: 18.

12. Studdiford J, Stonehouse A, Altshuler M, Rinzler E. The management of keloids: hands-on versus hands-off. J Am Board Fam Med 2008; 21: 149-52.
13. Kelly AP. Surgical treatment of keloids secondary to ear piercing. J Nati Med Assoc 1978; 70: 349.

14. Jones K, Fuller CD, Luh JY, et al. Case report and summary of literature: giant perineal keloids treated with post-excisional radiotherapy. BMC Dermatol 2006; 6: 7 .

15. Epstein E. Triamcinolon and keloids. West J Med 1980; 133: 257-258.

16. Darougheh A, Asilian A, Shariati F. Intralesional triamcinolone alone or in combination with 5-fluorouracil for the treatment of keloid and hypertrophic scars. Clin Exp Dermatol 2009; 34: 219-23.

17. Kalra A, Gupta S. Efficacy and safety of 5-fluorouracyl in the treatment of keloids. Dermatology 2002; 204: 130-2.

18. Wang XQ, Liu YK, Qing C, Lu SL. A review of the effectiveness of antimitotic drug injections for hypertrophic scars and keloids. Ann Plast Surg 2009; 63: 688-92.

19. Haurani MJ, Foreman K, Yang JJ, Siddiqui A. 5-Fluorouracil treatment of problematic scars. Plast Reconstr Surg 2009; 123: 139-48.

20. Bouzardi N, Davis SC, Nouri K. Laser treatment of keloids and hypertrophic scars. Int J Dermatol 2007; 46: 80-8.

21. Scrimali L, Lomeo G, Nolfo C, et al. Treatment of hypertrophic scars and keloids with fractional $\mathrm{CO}_{2}$ laser: a personal experience. J Cosmet Laser Ther 2010; 12: 218-21.

22. Toboła J, Witkowska-Toboła A, Włodarkiewicz A. Silicone gel in prevention of hypertrophic scars and keloids [Polish]. Derm Estet 2004; 6: 323-8.

23. Leshaw SM. Silicone use in keloids. West J Med 1994; 160: 363-4.

24. Sand M, Sand D, Boorboor P, et al. Combination of surgical excision and custom designed silicon pressure splint therapy for keloids on the helical rim. Head Face Med 2007; 3: 14.

25. Puri N, Talwar A. The efficacy of silicone gel for the treatment of hypertrophic scars and keloids. J Cutan Aesthet Surg 2009; 2: 104-6.

26. Hatamipour E, Mehrabi S, Hatamipour M, Ghafarian Shirazi HR. Effects of combined intralesional 5 -fluorouracil and topical silicone in prevention of keloids: a double blind randomized clinical trial study. Acta Med Iran 2011; 49: 127-30.

27. Louis DD, Garcia C. Pressure earring as an adjunct to surgical removal of earlobe keloids. Dermatol Surg 2010; 36: 726.

28. Ogawa R, Yoshitatsu S, Yoshida K, Miyashita T. Is radiation therapy for keloids acceptable? The risk of radiation-induced carcinogenesis. Plast Reconstr Surg 2009; 124: 1196-201.

29. Har-Shai Y, Brown W, Pallua N, Zouboulis CC. Intralesional cryosurgery for the treatment of hypertrophic scars and keloids. Plast Reconstr Surg 2010; 126: 1798-800.

30. Zieliński T. Treatment of the earlobes keloids with cryosurgery [Polish]. Prz Dermatol 2006; 94: 289-91.

31. Pierce HE. Cryosurgery for hypertrophic scars and keloids. A preliminary report. J Nat Med Assoc 1974; 66: 174-5.

32. Berman B, Harrison-Balestra C, Perez OA. Treatment of keloid scars post-shave excision with imiquimod $5 \%$ cream: a prospective, double-blind, placebo-controlled pilot study. J Drugs Dermatol 2009; 8: 455-8.

33. D'Andrea F, Brongo S, Ferraro G, et al. Prevention and treatment of keloids with intralesional verapamil. Dermatology 2002; 204: 60-2.

34. Xu SJ, Teng JY, Xie J, et al. Comparison of mechanisms of intralesional steroid, interferon or verapamil injection in the treatment of proliferative scars. Chin J Plas Surg 2009; 25: 37-40.

35. Davidson SP, Mess S, Kuaffman L, Al-Attar A. Ineffective treatment of keloids with interferon alpha-2b. Plast Reconstr Surg 2006; 117: 247-52. 
36. Viera MH, Caperton CV, Berman B. Advances in the treatment of keloids. J Drugs Dermatol 2011; 10: 468-80.

37. Iannello S, Milazzo P, Bordonaro F, Belfiore F. Low-dose enalapril in the treatment of surgical cutaneus hypertrophic scar and keloid - two case reports and literature review. Med Gen Med 2006; 8: 60.

38. Zhang GY, Yi CG, Li X, et al. Troglitazon supress transforming growth factor-beta1-induced collagen type I expression in keloid fibroblasts. Br J Dermatol 2009; 160: 762-70.

39. Santiago B, Gutierrez-Canas I, Dotor J, et al. Topical application of a peptide inhibitor of transforming growth factor beta1 ameliorates bleomycin-induced skin fibrosis. J Invest Dermatol 2005; 125: 450-5.

40. Ooi BN, Phan TT. Insights gained from the reverse engineering of gene networks in keloid fibroblasts. Theor Biol Med Model 2011; 8: 13 . 